\title{
Article
}

\section{Family-led rehabilitation in India (ATTEND)-Findings from the process evaluation of a randomized controlled trial}

Liu, Hueiming, Lindley, Richard, Alim, Mohammed, Felix, Cynthia, Gandhi, Dorcas BC, Verma, Shweta J, Tugnawat, Deepak K, Syrigapu, Anuradha, Ramamurthy, Ramaprabhu K, Pandian, Jeyaraj D, Walker, Marion, Forster, Anne, Hackett, Maree, Anderson, Craig S, Langhorne, Peter, Murthy, Gudlavalleti VS, Maulik, Pallab K, Harvey, Lisa A and Jan, Stephen

Available at http://clok.uclan.ac.uk/23714/

Liu, Hueiming, Lindley, Richard, Alim, Mohammed, Felix, Cynthia, Gandhi, Dorcas BC, Verma, Shweta J, Tugnawat, Deepak K, Syrigapu, Anuradha, Ramamurthy, Ramaprabhu K et al (2019) Family-led rehabilitation in India (ATTEND)_Findings from the process evaluation of a randomized controlled trial. International Journal of Stroke, 14 (1). ISSN 1747-4930

It is advisable to refer to the publisher's version if you intend to cite from the work. http://dx.doi.org/10.1177/1747493018790076

For more information about UCLan's research in this area go to http://www.uclan.ac.uk/researchgroups/ and search for < name of research Group>.

For information about Research generally at UCLan please go to http://www.uclan.ac.uk/research/

All outputs in CLoK are protected by Intellectual Property Rights law, including Copyright law. Copyright, IPR and Moral Rights for the works on this site are retained by the individual authors and/or other copyright owners. Terms and conditions for use of this material are defined in the policies page. 

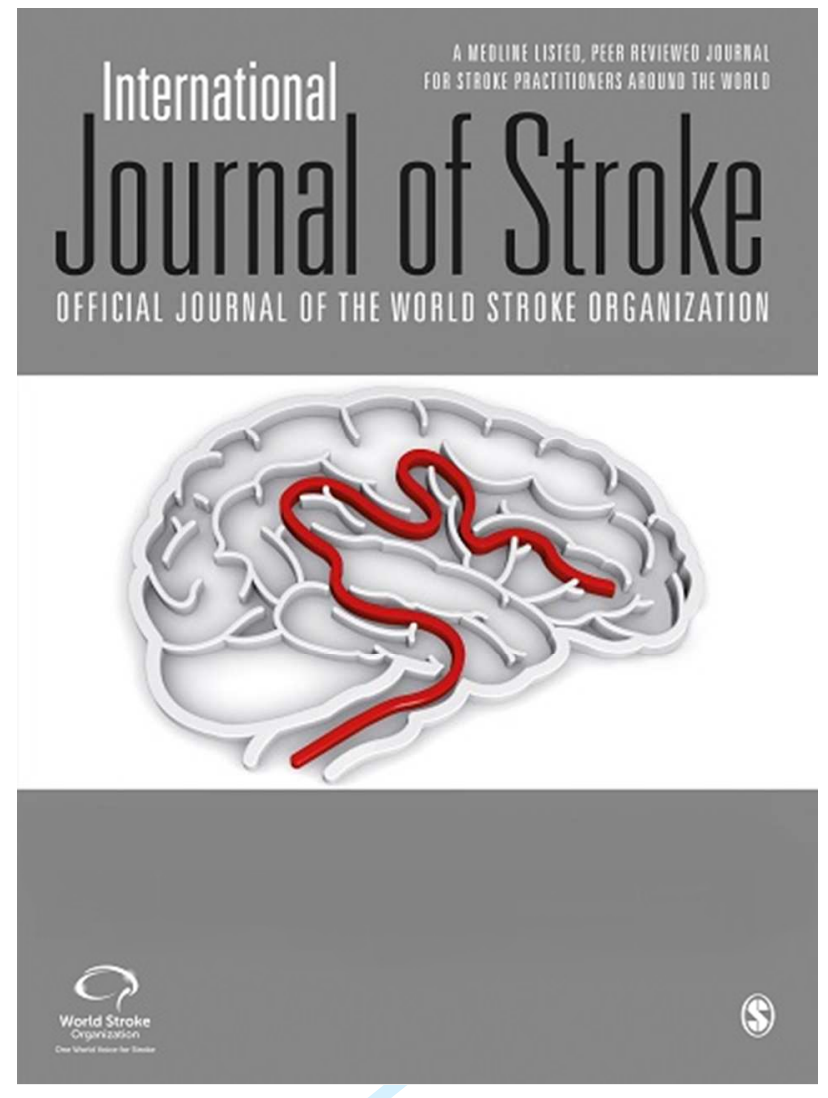

\section{Family-led rehabilitation in India (ATTEND) - findings from the Process Evaluation of a Randomised Controlled Trial}

\begin{tabular}{|r|l|}
\hline Journal: & International Journal of Stroke \\
\hline Manuscript ID & IJS-11-17-6053.R1 \\
\hline Manuscript Type: & Research \\
\hline Date Submitted by the Author: & 02-Apr-2018 \\
\hline Complete List of Authors: & $\begin{array}{l}\text { Liu, Hueiming; The George Institute for Global Health, Health Economics } \\
\text { and Process Evaluation Program; University of Sydney - Camperdown and } \\
\text { Darlington Campus, } \\
\text { Lindley, Richard; George Institute for Global Health, Neurological and } \\
\text { Mental Health } \\
\text { Alim, Mohammed; The George Institute for Global Health, India, } \\
\text { Felix, Cynthia; George Institute for Global Health, Hyderabad, India. } \\
\text { Gandhi, Dorcas; Christian Medical College, Ludhiana, Punjab, India, } \\
\text { Department of Physiotherapy } \\
\text { Verma, Shweta; Stroke Unit, Neurology } \\
\text { Tugnawat, Deepak; Indian Institute of Public Health } \\
\text { Syrigapu, Anuradha ; Indian Institute of Public Health }\end{array}$ \\
\hline
\end{tabular}




\begin{tabular}{|l|l|}
\hline & Ramamurthy, Ramaprabhu ; Medcare Physiotherapy and Rehabilitation \\
Centre & Pandian, Jeyaraj; Christian Medical College, Stroke Unit, Department of \\
Neurology & Walker, Marion; University of Nottingham, Rehabilitation and Ageing \\
Forster, Anne; University of Leeds and Bradford teaching Hospital NHS \\
Foundation Trust, Academic Unit of Elderly Care and Rehabilitation \\
Hackett, Maree; The George Institute for Global Health, Neurological \& \\
Mental Health Division, The University of Sydney; The University of Central \\
Lancashire, School of Health \\
Anderson, Craig; Royal Prince Alfred Hospital and University of Sydney, \\
The George Institute for International Health \\
Langhorne, Peter; University of Glasgow, Institute of Cardiovascular and \\
Medical Sciences \\
Murthy, G.V.S; Indian Institute of Public Health Hyderabad, \\
Maulik, Pallab; George Institute for Global Health India, Research and \\
Development; University of Oxford, The George Institute for Global Health \\
Harvey, Lisa; University of Sydney, Rehabilitation Studies Unit, Northern \\
Clinical School, Sydney School of Medicine \\
Jan, Stephen; The George Institute for Global Health, University of Sydney, \\
\hline Keywords: \\
$\begin{array}{l}\text { Qualitative research, Randomised Controlled Trial, India, Process } \\
\text { Evaluation, Rehabilitation, Stroke }\end{array}$ \\
\hline
\end{tabular}

\section{SCHOLARONE ${ }^{\text {" }}$}

Manuscripts 
Family-led rehabilitation in India (ATTEND) - findings from the Process Evaluation of a $\underline{\text { Randomised Controlled Trial }}$

Authors:

*Hueiming Liu ${ }^{1,2,3}$ MIPH (hliu@georgeinstitute.org.au)

Richard Lindley ${ }^{1,2}$ MD FRACP (닉ley@georgeinstitute.org.au),

Mohammed Alim ${ }^{4}$ M. Pharmacy (malim@georgeinstitute.org.in),

Cynthia Felix ${ }^{4}$ MD (cfelix@georgeinstitute.org.in),

Dorcas BC Gandhi ${ }^{5}$ MPT (dorcasattend@gmail.com),

Shweta J Verma ${ }^{5}$ MSc (sjvmv1947@,gmail.com),

Deepak K Tugnawat ${ }^{6}$ BPT (deepak.k@iiphh.org),

Anuradha Syrigapu ${ }^{6}$ BDS (radhasyrigapu@gmail.com),

Ramaprabhu K Ramamurthy ${ }^{7}$ MPT (physioram2002@gmail.com)

Jeyaraj D Pandian ${ }^{5}$ DM FRACP (jeyarajpandian@hotmail.com)

Marion Walker ${ }^{8} \mathrm{PhD}$ (marion.walker@nottingham.ac.uk)

Anne Forster ${ }^{9,8} \mathrm{PhD}$ (

Maree L Hackett ${ }^{1,2,3,11}$ PhD (․ㅡhackett@georgeinstitute.org.au $)$

Craig S Anderson ${ }^{1,2,3} \mathrm{PhD}$ (canderson@georgeinstitute.org.au)

Peter Langhorne ${ }^{12} \mathrm{PhD}$ (Peter.Langhorne@glasgow.ac.uk)

Gudlavalleti VS Murthy ${ }^{6}$ MD MSc (murthy.gvs@iiphh.org)

Pallab K Maulik ${ }^{4,10} \mathrm{PhD}$ (pmaulik@georgeinstitute.org.in)

Lisa A Harvey² PhD (lisa.harvey@,sydney.edu.au $)$

Stephen Jan ${ }^{1,2,3} \mathrm{PhD}$ (sjan@georgeinstitute.org)

Author Affiliations 
1 The George Institute for Global Health, Sydney, Australia.

2 University of Sydney, Sydney, Australia.

3 University of New South Wales, Sydney, Australia

4 George Institute for Global Health, Hyderabad, India.

5 Christian Medical College, Ludhiana, Punjab, India.

6 Indian Institute of Public Health, Hyderabad, India.

7 Medcare Physiotherapy and Rehabilitation Centre, Dubai, United Arab Emirates.

8 University of Nottingham, Nottingham, United Kingdom.

9 University of Leeds, West Yorkshire, England, United Kingdom.

10 The George Institute for Global Health, Oxford University, United Kingdom.

11University of Central Lancashire, Preston, United Kingdom

12 University of Glasgow, United Kingdom

*Corresponding Author

Address: PO Box M201, Missenden Rd, NSW 2050 Australia

Email: hliu@georgeinstitute.org.au 


\section{ABSTRACT}

Background: Training family carers to provide evidence-based rehabilitation to stroke patients could address the recognised deficiency of access to stroke rehabilitation in lowresource settings. However, our randomised controlled trial in India (ATTEND), found that this model of care was not superior to usual care alone.

Aims: This process evaluation aimed to better understand trial outcomes through assessing trial implementation and exploring patients', carers' and providers' perspectives.

Methods: Our mixed methods study included process, healthcare use data and patient demographics from all sites; observations and semi-structured interviews with participants (22 patients, 22 carers and 28 health providers) from 6 sampled sites.

Results: Intervention fidelity and adherence to the trial protocol was high across the 14 sites, however, early supported discharge (an intervention component) was not implemented.

Within both randomised groups some form of rehabilitation was widely accessed. ATTEND stroke coordinators provided counselling and perceived that sustaining patients' motivation to continue with rehabilitation in the face of significant emotional and financial stress as a key challenge. The intervention was perceived as an acceptable community-based package with education as an important component in raising the poor awareness of stroke. Many participants viewed family-led rehabilitation as a necessary model of care for poor and rural populations who could not access rehabilitation.

Conclusion: Difficulty in sustaining patient and carer motivation for rehabilitation without ongoing support, and greater than anticipated access to routine rehabilitation may explain the lack of benefit in the trial. Nonetheless family-led rehabilitation was seen as a concept worthy of further development. 
Word count: 4730 (250 (abstract), 3579 (main text), 926 (Ref))

Key words: Qualitative research, Stroke, Rehabilitation, Randomised Controlled Trial,

Process Evaluation, India

2 Figures and 4 supplementary files 


\section{$\underline{\text { INTRODUCTION }}$}

The global burden of stroke is increasing, and in low and middle income countries (LMICs) tends to affect individuals at an earlier age than in high income countries, resulting in a significant societal burden. (1) India is in the midst of a major epidemiological transition, with an increasing stroke prevalence but limited accessibility to affordable and high quality rehabilitation services. (2-4) Task shifting through family-led rehabilitation may offer a potential sustainable solution in India and other LMICs. (5-7)

The ATTEND Trial (fAmily led rehabiliTaTion trial aftEr stroke trial in INDia) was a randomised controlled trial (RCT), which recruited 1250 patients across 14 hospitals. (8) The intervention involved a physiotherapist (known in the trial as a 'stroke coordinator') training a family carer to deliver a basic evidence-based rehabilitation intervention tailored to the Indian context. (9) The intervention had five main components including education, positioning/early mobilisation, early supported discharge planning, joint goal setting with each patient and carer, and task-orientated training. Stroke coordinators trained family carers to provide rehabilitation during hospital admission and at subsequent home visits (maximum of six) in the first two months. A culturally appropriate intervention manual documenting intervention components was provided to the patient during the first home visit. Patients and carers were followed up at three and six months by an assessor blinded to intervention allocation and its content. The recently published results indicated that this model of 'task shifting' rehabilitation to family carers compared to usual care alone did not achieve a benefit, as measured by a decrease in death or dependency. (8)

In the wake of ATTEND's neutral results, important questions remain about the future of family-led rehabilitation and uncertainty as to what transpired on the ground: was the trial result due to failure of the intervention or of trial implementation, or of both? (10) 


\section{$\underline{\text { AIMS }}$}

Our process evaluation was conducted alongside the ATTEND RCT to explore how, for whom and why this complex intervention had (or did not have) an impact. Our a priori aims were: (1) To explore if the ATTEND trial was conducted rigorously; (2) To understand providers', patients' and carers' perspectives of the perceived effect of the care they received or delivered; (3) To explore if the results are likely to be generalisable, scalable and sustainable through exploring stakeholders' experiences of the intervention and its perceived impact. (11)

\section{$\underline{\text { METHODS }}$}

The process evaluation methods were pre-specified in detail in a published protocol. (11) We present a summary here.

\section{Process evaluation framework}

The design of our mixed methods process evaluation (Figure 1) was informed by two theoretical frameworks covering different aspects of the evidence generation-to-practice translation continuum. The RE-AIM framework incorporates concepts of Reach, Effectiveness, Adoption, Implementation and Maintenance to address questions of generalisability and translation into practice. (12) The Realist framework highlights potential mechanisms of action within contextual factors and is pertinent for complex interventions such as ATTEND. $(13,14)$ Our hypothesised causal mechanisms are stated explicitly in Figure 1 of how this intervention may impact upon proposed trial outcomes.

\section{$\underline{\text { Data sources }}$}

We collected quantitative process data on intervention delivery, patient demographics and healthcare use data across all 14 sites. Qualitative data included semi-structured interviews with 28 health providers (seven neurologists, eight stroke coordinators, eight blinded assessors, four physiotherapists responsible for usual care and one clinical nurse), 22 patients 
and 22 carers ( 11 usual care and 11 intervention arm dyads), observations (using a standardised template), and documentary analysis from six purposively sampled sites to enable a cross-section of geographical locations and types of institutions. (15) Patient and carer dyads were purposively sampled by patient gender and study arm, and interviews at the hospital were conducted shortly after their six months follow-up visit by interviewers who had no interactions with the dyads before the interviews. Questions covered patients' health care journey, components of the intervention and contextual factors. Healthcare providers were interviewed (by AM, HL, RL, AF, MW) in English and all patients and carers were interviewed (by AM, DG, CF, SV) in their local Indian languages where possible. There were two sites in which interpreters were used to communicate in local languages. All interviews were audio recorded. The interviews lasted on average 30 minutes and all were transcribed verbatim by professional transcribers and translators. We received ethical approval for the conduct of the health provider interviews from each study site, with one site not granting approval for patient and carer interviews and as such patient and carer interviews were only conducted at five sites. (Supplementary file 1 provides key participant characteristics and additional illustrative quotes.)

\section{$\underline{\text { Analysis }}$}

Iterative thematic analysis of the qualitative data was conducted by the team blinded to the RCT outcomes. (14) NVivo software was used to manage the data. A coding tree based on the main headings of our process evaluation framework and line by line coding was created by HL. (Supplementary file 2) As pre-specified in the process evaluation protocol, triangulation of the qualitative findings was then conducted with baseline demographics, healthcare use and primary and secondary outcomes, and then systematically analysed against our hypothesised causal mechanisms according to the Realist configuration of ContextMechanisms-Outcomes. (14) 


\section{$\underline{\text { RESULTS }}$}

Our results are summarised and depicted in Figure 2.

\section{(1) Trial fidelity: trial implementation and intervention fidelity}

Reach and Recruitment:

We assessed ATTEND baseline data to determine whether recruited patients were representative of the broader Indian stroke population and found that ATTEND patients had a higher level of education and monthly household income than the national population average. $(8,16)$ The limited feasibility for the ATTEND stroke coordinators to follow up patients living more than $50 \mathrm{~km}$ from the 14 participating mainly urban RCT sites (4 governmental central institutes, 4 Christian Mission and Academic Institutions, and 6 private corporate hospitals) may have contributed to this difference.

We explored healthcare use to determine the level of access to healthcare and rehabilitation by the patients recruited and found that at 6 months, most of the patients in the intervention and usual care arm continued non-trial conventional therapy i.e. either rehabilitation or medications. In both groups, about $45 \%$ reported incurring charges for outpatient rehabilitation therapy at 3 months, and about $30 \%$ at 6 months.

We synthesised our findings to better understand routine care. We found that the different types of hospitals had differing costs of treatment and available routine care in terms of stroke unit guidelines, specialist staffing, and presence of multidisciplinary outpatient clinics. In particular, we noted at 3 hospitals routine care comprised of outpatient clinics with rehabilitation provided by physiotherapists who described training family carers as part of routine care. Three hospitals had multidisciplinary outpatient clinics -including one corporate hospital which had established links (e.g. conducting capacity building workshops) with free- 
lance private physiotherapists in the community who could provide rehabilitation in patients' homes.

\section{Unblinding:}

Assessors reported any unblinding to the project manager. The reports showed that unblinding occurred in 5.3\% in the intervention group, and $3.3 \%$ in the usual care group $(p=0.09)$. An example of 'unblinding' included inadvertent incidents such as the intervention manual being seen on the table at home.

\section{Contamination:}

The potential for 'contamination' of usual care patients due to lack of space, or curtains around beds to conduct the intervention in privacy at smaller hospitals was noted during observation visits.

Intervention Fidelity:

The activity logs highlighted that the ATTEND components of goal setting, gait training and functional mobility training were implemented as per protocol. (8) Intervention dose, as indicated by duration of therapy provided, showed an average hospital training time of 2.96 hours, (SD 1.56, median 2.92), mean home training time of 3.07 hours (SD 1.69, median 2.75). Patients and carers self-reported 17.8 hours (SD 21.56) of activities in the first 30 days following hospital discharge. Activity logs of the usual care physiotherapists show that similar non-trial rehabilitation care was provided both randomised groups. Early supported discharge as a component of the ATTEND intervention was not achieved, with both groups having a similar length of hospital stay. (8)

(2) For whom, how and why? To understand providers', patients' and carers' perspectives of the perceived effect of the care they received / delivered. 
Health providers stated that the early supported discharge component of ATTEND was welcomed in concept due to potential cost savings for patients and earlier release of bedspace for the hospital. However, several neurologists described early supported discharge was not implemented due to an inadequate number of hospital beds, which resulted in patients being discharged as early as possible, or even not admitted for care, irrespective of enrolment in ATTEND. At one hospital it was estimated that $40 \%$ of patients discharged themselves from the hospital against medical advice reportedly due to the unaffordable costs of hospitalisation. With the exception of one stroke coordinator, most health providers indicated that the ATTEND intervention was not factored into the discharge planning (i.e. time of discharge) for patients in the intervention group.

\section{Stroke education is needed}

Education about stroke, stroke risk factors and the value of rehabilitation was provided to patients and carers in the intervention arm. A carer of an intervention patient described how community members had initially expected his father to die but they have since seen that “(his father) can walk on his own...and said because of exercises only he has improved that much." Indeed, carers and patients in the intervention arm described a deeper understanding of the pathophysiology of stroke and a greater confidence in recovery. Most of the health providers stressed that addressing the low level of community health literacy was a priority as there was often poor management of risk factors in the community (as seen by the relatively young age of participants compared to high income countries) and delayed presentation to hospital.

\section{ATTEND is an acceptable model of care}

Many stroke coordinators and intervention carers indicated that joint goal setting with patient and carer was a key component of ATTEND and that this process was crucial in the patients' 
recovery. For example, an intervention patient described how "half of my body had become useless. But I am thankful to my children, within fifteen days they helped me...I just stood like that, with the support of their shoulders...then they instructed me to do things." Some stroke coordinators commented favourably on the multi-disciplinary aspect of ATTEND (e.g. components of occupational and speech therapy) and the active rehabilitation (e.g. taskspecific training), as compared to the passive physiotherapy they previously provided. This was evident in the activity forms, which showed a greater emphasis (as indicated by percentage of time spent) on goal setting and functional task training provided by the stroke coordinators as compared to usual care physiotherapists. (8)

Stroke coordinators liked the training of a nominated caregiver in ATTEND which encouraged continuity of the care provided to the patient. The ATTEND trial intervention manual and videos were key resources for the patients and carers. The stroke coordinators and a few neurologists highlighted that home visits added valuable contextual information for functional training. Moreover, home visits were preferred by the patients and carers as this removed the cost and travel barriers faced when attending hospital follow-up visits.

\section{Sustaining patient and carer motivation was a key challenge}

Several of the stroke coordinators reported that counselling was critical in the early stages post stroke to maintain patient motivation and overcome their initial despair. A few of the stroke coordinators suggested that more visits than the trial goal of three to six visits would be necessary to sustain patients' and carers' motivation to persist with rehabilitation. A stroke coordinator observed that individual patients' will to recover was affected by their gender roles, he described that "males don't have much patience as they have to go earn for his family... They don't want to spend much time on bed... Usually more females are housewives, and some (lose) their hopes from getting up from bed." The importance of this observation is 
highlighted when triangulated with the finding that men appeared to benefit more than women in the trial. (8) A doctor described that carers' motivation depended on individual family circumstances regardless of education and literacy status, and that "it just depends on how stressed the family is...there are other patients who are totally illiterate but they are so willing to learn this, so willing to do it for their relatives...I mean every patient...their situation is different." Additionally, managing bowel incontinence, and patients' pain were also described as particularly trying for carers.

Financial stress due to loss of income and cost of treatment was iterated by many of the participants as impacting on the complexities of the patients' and carers' relationship, mental health, and motivation. For example, a female carer described selling her jewellery to tide their family through financial difficulties and saw little hope for the future. A stroke coordinator observed that "if the stroke affects a middle-aged man, the family is [in] a disaster" due to the decrease in household income which could lead to poverty and reduce children's educational opportunities.

(3) To explore if the results are likely to be generalisable, scalable and sustainable through exploring stakeholders' experiences of the intervention and its perceived impact. ATTEND is a sustainable model of care especially for those with limited access to rehabilitation

Many participants perceived that ATTEND would be ideal for the poor and those in rural settings who could not access acute treatment and rehabilitation due to distance to services and high cost. A physician cited ATTEND as a proof of concept of a model of care for her region where there were "no physiotherapists out there who will go to the (villagers') homes or whom patients can go to and get help." She described that "even before this study started, 
we were giving the relatives the education that they needed and trying to teach them to help their patients..."

When affordability was an issue, carers would seek advice from the community on managing rehabilitation at home. The source of such information included local physiotherapists, other community members who had recovered from stroke or paralysis, and traditional masseurs. For example, a control arm carer described how they could not afford the INR300 per physiotherapist visit and had pleaded with him to "please teach us. As our condition is such we will do it ourselves. After that we do on our own. We are still doing it."

While ATTEND was delivered free in the trial, many of the intervention patients and carers indicated they would be willing to pay a fee and would recommend this treatment to others indicating an assumption that the intervention was effective. Indeed, two ATTEND carers described providing rehabilitation for other stroke patients in their community. Some health providers suggested incorporating the ATTEND intervention into their routine practice at the stroke unit. A neurologist suggested conducting ATTEND training workshops for the freelance physiotherapists practicing in the community. For areas with limited access to service providers, two neurologists suggested community-based models of care as potentially feasible. One described the potential to have Accredited Social Health Activists (ASHAs) trained and certified to provide the ATTEND intervention. However, other neurologists and physiotherapists stressed that such community-based models would require significant upskilling, supervision and monitoring so as to prevent exploitation by other nonlicensed/untrained providers.

\section{DISCUSSION}

Our data confirm that ATTEND was a rigorously conducted trial of an intervention designed to balance existing best practice rehabilitation with local norms and economic constraints. $(8$, 
9) Furthermore, the process evaluation was conducted with a pre-specified protocol and conceptual framework. (11) We found that intervention fidelity and adherence to the protocol was consistently high across sites with the exception that early supported discharge was welcomed in concept but not widely implemented. Using the Realist framework of ContextMechanisms-Outcomes, we have identified two reasons for why we did not achieve the expected trial result (as depicted in Figure 2). (13) First, whilst we had hypothesised that a family carer providing rehabilitation would represent a step-up in access to care (4), our contextual findings suggest that many patients from both randomised groups already had access to rehabilitation which included rehabilitation that family members had been trained to deliver. Second, whilst we confirmed our hypothesis that a key mechanism of ATTEND was joint goal setting, we found that sustaining behaviour change for patients and carers in the face of significant emotional and financial pressures was a challenge. We also noted that stroke coordinators spent time counselling the patients and carers (which was not outlined in the protocol), and this may have decreased the time available for teaching physical training and task specific activities. Thus, the failure to reduce death and dependency over usual care is likely to be due to difficulties in initiating rehabilitation training because of the counselling needs of the patient, ongoing challenges in sustaining patient and carer motivation; and the higher than expected levels of rehabilitation may have diluted any potential benefits of death and dependency (as measured by the modified Rankin scale) and the other secondary outcome measures (such as patient mood, quality of life and carer strain).

Our findings also imply that in regions where rehabilitation is not accessible, the concept of task-shifting to community members (e.g. ASHA) and family carers has a lot of support. (2, $4,5)$ The challenge lies in how the intervention could be adapted in the future for such settings, whilst ensuring that the training is standardised and certified. (17) Given the high 
penetration of mobile telephones in India, mobile technology-enabled training and rehabilitation could be tried in rural settings with remote monitoring provided by specialists through video-links i.e. telemedicine. $(18,19)$ In addition, community-based prevention strategies including e-health may be beneficial in addressing the poor awareness of stroke, its risk factors and treatment as highlighted in this and other studies. (20-22) Critically, future versions of the family-led rehabilitation model will require increases in training intensity, duration and dose to encourage sustained behaviour. The timing of these functions should be informed by the patients' and carers' capacity to assimilate information. As such, training to provide rehabilitation may be more effective when patients and family carers are past the acute stroke crisis stage of intense shock and grief. $(5,23)$ Moreover, given the impact of financial stress upon patient and carers, we will further examine the economic data from ATTEND to identify critical variables contributing to out of pocket costs and household financial catastrophe.

A limitation of this process evaluation is the generalisability of our qualitative findings based on six sampled sites. Moreover, participants who agreed to be interviewed may have been biased positively to ATTEND, and we may have missed contradictory insights. However, our purposive sampling by hospital characteristics, inclusion of usual care dyads and triangulation with other data sources increases the validity of the findings. (24) The use of the theoretical frameworks especially around the topics of Reach and Maintenance, and the Context-Mechanisms-Outcomes configuration was valuable in synthesising our findings. In addition, describing the initial hypothesised causal mechanisms of the intervention provided a systematic way of analysing the data and exploring reasons for the trial outcomes. (14) In retrospect, more formative work by conducting qualitative interviews (with patients, carers and implementers) alongside the single centre pilot feasibility trial may have identified some finer details of implementation (e.g. timing for patients and carers to assimilate information) 
and dosing (e.g. number of optimal visits) that may have promoted efficacy of the ATTEND intervention. (9) Such in-depth exploratory work alongside pilot trials could inform key modifications needed to improve complex interventionssuch as ATTEND).(25, 26)

\section{CONCLUSIONS}

Our findings indicate that family-led rehabilitation is worthy of further development especially for the poor and rural populations in India. Future family-led rehabilitation should include behavioural change and sustainability components, with an increase in the intensity and duration of effective training modules; whilst maintaining cost and logistic feasibility for populations with limited access to rehabilitation. The ATTEND trial and process evaluation is the first step for the ATTEND collaboration in further developing patient-centred rehabilitation models of care needed to address the rising burden from stroke in India and other LMICs. (27) 
Acknowledgements:We thank the participants for their valuable insights; Mr Qiang Li for providing the analysis of the healthcare use data; and all the stroke coordinators, blinded assessors and trial management team in the successful completion of the ATTEND RCT.

Funding Statement: This study is funded by the National Health and Medical Research Council of Australia (Project grant no APP1045391). PM is funded by an Intermediate Career Fellowship of Wellcome Trust-Department of Biotechnology India Alliance. MLH is funded by a National Heart Foundation Future Leader Fellowship, SJ is funded by a NHMRC Senior Research Fellowship. CA is funded by a NHMRC Senior Principal Research Fellowship. HL is funded by a NHMRC postgraduate scholarship.

Competing interests: The authors declare that they have no competing interests.

Authors' contributions: JDP suggested the original ATTEND trial idea and the steering committee (RL,GVSM,PKM,PL,LAH,MLH,MW,AF,BRS,CSSA and SJ) designed the trial with a process evaluation in mind. HL developed the process evaluation protocol with significant contribution in its design, data collection and analysis from the trial management team (MA, CF, DBCG, SJV, DKT, AS and RKR). HL led the analysis and drafted the manuscript. All authors contributed to the revisions and approve the manuscript. 


\section{$\underline{\text { References }}$}

1. Feigin VL, Forouzanfar MH, Krishnamurthi R, Mensah GA, Connor M, Bennett DA, et al. Global and regional burden of stroke during 1990-2010: findings from the Global Burden of Disease Study 2010. Lancet. 2014 Jan 18;383(9913):245-54. PubMed PMID: 24449944. Pubmed Central PMCID: 4181600.

2. Kalkonde YV, Deshmukh MD, Sahane V, Puthran J, Kakarmath S, Agavane V, et al. Stroke Is the Leading Cause of Death in Rural Gadchiroli, India: A Prospective Community-Based Study. Stroke; a journal of cerebral circulation. 2015 Jul;46(7):1764-8. PubMed PMID: 25999388.

3. Biswas T. India unveils plans to improve stroke research and care. Lancet. 2013 Jan 19;381(9862):190. PubMed PMID: 23346589.

4. Pandian JD, Sudhan P. Stroke epidemiology and stroke care services in India. Journal of stroke. 2013 Sep;15(3):128-34. PubMed PMID: 24396806. Pubmed Central PMCID: 3859004.

5. Kamalakannan S, Gudlavalleti Venkata M, Prost A, Natarajan S, Pant H, Chitalurri N, et al. Rehabilitation Needs of Stroke Survivors After Discharge From Hospital in India. Archives of physical medicine and rehabilitation. 2016 Sep;97(9):1526-32 e9. PubMed PMID: 26944710.

6. Pandian JD, Srikanth V, Read SJ, Thrift AG. Poverty and stroke in India: a time to act. Stroke; a journal of cerebral circulation. 2007 Nov;38(11):3063-9. PubMed PMID: 17954905.

7. Prakash V, Shah MA, Hariohm K. Family's presence associated with increased physical activity in patients with acute stroke: an observational study. Brazilian journal of physical therapy. 2016 Jun 16. PubMed PMID: 27333484.

8. Group AC. Family-led rehabilitation after stroke in India (ATTEND): a randomised controlled trial. Lancet. 2017 Jun 27. PubMed PMID: 28666682.

9. Pandian JD, Felix C, Kaur P, Sharma D, Julia L, Toor G, et al. FAmily-Led RehabiliTaTion aftEr Stroke in INDia: the ATTEND pilot study. International journal of stroke : official journal of the International Stroke Society. 2015 Jun;10(4):609-14. PubMed PMID: 25753445.

10. Kwakkel G, van Wegen EEH. Family-delivered rehabilitation services at home: is the glass empty? Lancet. 2017 Jun 27. PubMed PMID: 28666681.

11. Liu H, Lindley R, Alim M, Felix C, Gandhi DB, Verma SJ, et al. Protocol for process evaluation of a randomised controlled trial of family-led rehabilitation post stroke (ATTEND) in India. BMJ Open. 2016 Sep 15;6(9):e012027. PubMed PMID: 27633636. Pubmed Central PMCID: 5030603.

12. Gaglio B, Phillips SM, Heurtin-Roberts S, Sanchez MA, Glasgow RE. How pragmatic is it? Lessons learned using PRECIS and RE-AIM for determining pragmatic characteristics of research. Implement Sci. 2014;9:96. PubMed PMID: 25163664. Pubmed Central PMCID: 4243945.

13. Pawson R, Tilley, N. Realistic Evaluation: Sage; 1997.

14. Moore GF, Audrey S, Barker M, Bond L, Bonell C, Hardeman W, et al. Process evaluation of complex interventions: Medical Research Council guidance. BMJ. 2015;350:h1258. PubMed PMID: 25791983. Pubmed Central PMCID: 4366184.

15. Patton MQ. Qualitative Research \& Evaluation Methods. 3rd Edition ed2002.

16. World Health Organisation . India WHO Statistical profile 2013. Available from: http://www.who.int/gho/countries/ind.pdf?ua=1.

17. Khan FR, Vijesh PV, Rahool S, Radha AA, Sukumaran S, Kurupath R. Physiotherapy practice in stroke rehabilitation: a cross-sectional survey of physiotherapists in the state of Kerala, India. Topics in stroke rehabilitation. 2012 Sep-Oct;19(5):405-10. PubMed PMID: 22982827.

18. Srivastava PV, Sudhan P, Khurana D, Bhatia R, Kaul S, Sylaja PN, et al. Telestroke a viable option to improve stroke care in India. International journal of stroke : official journal of the International Stroke Society. 2014 Oct;9 Suppl A100:133-4. PubMed PMID: 25042038.

19. Sureshkumar K, Murthy G, Natarajan S, Naveen C, Goenka S, Kuper H. Evaluation of the feasibility and acceptability of the 'Care for Stroke' intervention in India, a smartphone-enabled, carer-supported, educational intervention for management of disability following stroke. BMJ Open. 2016;6(2):e009243. PubMed PMID: 26839011. Pubmed Central PMCID: 4746451. 
20. Menon B, Swaroop JJ, Deepika HK, Conjeevaram J, Munisusmitha K. Poor awareness of stroke--a hospital-based study from South India: an urgent need for awareness programs. Journal of stroke and cerebrovascular diseases : the official journal of National Stroke Association. 2014 Sep;23(8):2091-8. PubMed PMID: 25113082.

21. Das S, Hazra A, Ray BK, Ghosal M, Chaudhury A, Banerjee TK, et al. Knowledge, attitude, and practice in relation to stroke: A community-based study from Kolkata, West Bengal, India. Annals of Indian Academy of Neurology. 2016 Apr-Jun;19(2):221-7. PubMed PMID: 27293334. Pubmed Central PMCID: 4888686.

22. Praveen D, Patel A, Raghu A, Clifford GD, Maulik PK, Mohammad Abdul A, et al. SMARTHealth India: Development and Field Evaluation of a Mobile Clinical Decision Support System for Cardiovascular Diseases in Rural India. JMIR mHealth and uHealth. 2014;2(4):e54. PubMed PMID: 25487047. Pubmed Central PMCID: 4275493.

23. Lutz BJ, Young ME, Cox KJ, Martz C, Creasy KR. The crisis of stroke: experiences of patients and their family caregivers. Topics in stroke rehabilitation. 2011 Nov-Dec;18(6):786-97. PubMed PMID: 22436315. Pubmed Central PMCID: 3822775.

24. Curry LA, Nembhard IM, Bradley EH. Qualitative and mixed methods provide unique contributions to outcomes research. Circulation. 2009 Mar 17;119(10):1442-52. PubMed PMID: 19289649.

25. Fletcher A, Jamal F, Moore G, Evans RE, Murphy S, Bonell C. Realist complex intervention science: Applying realist principles across all phases of the Medical Research Council framework for developing and evaluating complex interventions. Evaluation. 2016 Jul;22(3):286-303. PubMed PMID: 27478401. Pubmed Central PMCID: 4946011.

26. Chatterjee S, Chowdhary N, Pednekar S, Cohen A, Andrew G, Andrew G, et al. Integrating evidence-based treatments for common mental disorders in routine primary care: feasibility and acceptability of the MANAS intervention in Goa, India. World psychiatry : official journal of the World Psychiatric Association. 2008 Feb;7(1):39-46. PubMed PMID: 18458786. Pubmed Central PMCID: 2359726.

27. Pandian JD, Liu H, Gandhi DB, Lindley RI. Clinical stroke research in resource limited settings: Tips and hints. International journal of stroke : official journal of the International Stroke Society. 2017 Jan 1:1747493017743798. PubMed PMID: 29148963. 


\section{Figures}

Figure 1: The ATTEND process evaluation framework. Process evaluation components are highlighted in blue. *Reproduced with permission. (11)

Figure 2: Summary of the process evaluation findings (in blue italics) as compared against our hypothesised contextual assumptions and causal mechanisms (in non-italics) in impacting upon the outcomes of the RCT.

$\underline{\text { Supplementary files }}$

Supplementary file 1: Participant characteristics and Illustrative quotes.

Supplementary file 2: Coding Tree

Supplementary file 3: CONSORT statement (main trial paper)

Supplementary file 4: COREQ checklist 
Context- What are the contextual factors that are different across sites? (i.e socio- economic factors, pre-stroke/stroke/post stroke factors e.g accessibility of outpatient rehabilitation services, availability of below the poverty line insurance policies, availability and affordability of medications) What is part of usual care post stroke at each site? (c,d)

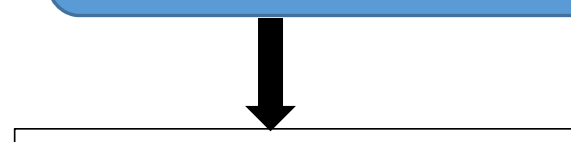

Description of ATTEND intervention components which address the assumed causal mechanisms contributing to the stroke burden in India

Poor awareness of stroke and

effectiveness of stroke rehabilitation

ATTEND: Education is provided to patient and carers on stroke

Limited resources and staffing to provide repetitious and active practice for the patients especially upon discharge.

Limited accessibility to multi-disciplinary stroke rehabilitation team.

ATTEND: Focus on gait training,

functional activities, home based care,

with inclusion of communication

strategies. Need for goal setting to

foster independence.

Post stroke care is provided mainly by

family members with limited knowledge and training

ATTEND: Joint goal setting with caregiver and patient; and training of the carer of suitable exercises.

Cost to outpatient care is a barrier for stroke rehabilitation

ATTEND: Early supported discharge to decrease costs to the system and family and also to enable early patient rehabilitation at home and foster independence.

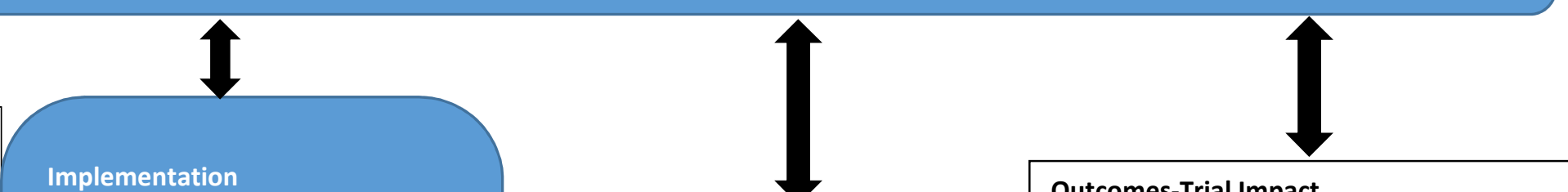

\section{Outcomes-Trial Impact}

Effectiveness/Outcomes- What is the primary

outcome measured at 3 months and at 6

months? Is there a difference? What are the secondary outcomes e.g hospital length of stay,

\section{Implementation}

Reach- How are the sites recruited? What is the burden of stroke in the area? What is the representativeness of the sites in providing care to stroke patients? How are the patients recruited? Are they representative of our target patient population? How many agreed or did not and why? (a, c, d)

Fidelity and Dose - Is the intervention delivered according to protocol at all the sites? Is there any contamination of the intervention to the control arm? $(a, b, c, d, e, f)$ there any barriers and facilitators to this process? $(d, e, f)$

Costs considerations- What is the cost of training and site visits? (a) What are intervention to patients? (b) barriers \& facilitators to trial implementation? (a, d, e, f)
Adoption- How is intervention incorporated into care provided? Are the costs of usual care and the

Implementation- What are some
Mechanisms of Impact

Effectiveness- How, why and for whom does the

intervention work? What are the health providers, patients and carers' experiences and attitudes towards the intervention and what they think the causal mechanisms are? $(e, d)$

Maintenance - Is this program generalizable, scalable and sustainable through exploring stakeholders' (hospital stroke unit staff, providers, patients and carers) experiences of the intervention and its perceived impact. Is there a plan in place to continue this intervention post-trial? $(e, f)$ caregiver burden? (b)

Economic evaluation- What is the cost effectiveness of this intervention from a societal and health systems perspective? (b)

Maintenance/ Long term impact - Is the family led rehabilitation model of care sustainable and can it lead to a reduction in the burden for stroke patients and to the health system? (g)

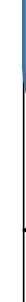

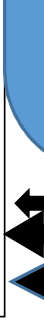




\section{Context}

1)Poor awareness of stroke and effectiveness of stroke rehabilitation

Need to address this was highlighted by participants

2) Limited resources and staffing to provide repetitious and active practice for the patients especially upon discharge. Limited accessibility to multi-disciplinary stroke rehabilitation team.

Availability of a range of rehabilitation services available at the hospitals recruited though participants highlighted limited accessibility and affordability to multidisciplinary rehabilitation in rural areas.

3) Post stroke care is provided mainly by family members with limited knowledge and training

Training is provided to the usual care family members at outpatient clinics post discharge and private physiotherapists were available in the community.

4) Cost to outpatient care is a barrier for stroke rehabilitation.

Barrier to patient engagement for rehabilitation was due to travel and treatment costs, and loss of family income.

\section{Mechanisms of impact}

1)Education is provided to patient and carers on stroke

ATTEND trial has raised the awareness in the community about stroke risk factors and the value of rehabilitation.

2)Focus on gait training, functional activities, home based care, with inclusion of communication strategies. Need for goal setting to foster independence.

ATTEND is an acceptable model of care especially for regions of limited accessibility.

3)Joint goal setting with caregiver and patient; and training of the carer of suitable exercises

In addition to the joint goal setting, the relationship between trial staff and patient and family seemed protective against poor mental status but maintaining motivation post intervention could be challenging.

4) Early supported discharge to decrease costs to the system and family and also to enable early patient rehabilitation at home and foster independence.

While early supported discharge is welcomed in concept, implementation depended on hospitals bed pressure, and patient factors (such as affordability of hospitalisation).

\section{Outcomes from the ATTEND RCT (8)}

Our baseline demographics which was 857 male, and 413 female with a mean age of 57.7 years old, and a majority of $89.7 \%$ married; had a high risk factor profile of $73.9 \%$ hypertension, $43.9 \%$ diabetes mellitus, $24.3 \%$ smoking, and $26.8 \%$ alcohol use, with a $18 \%$ of recurrent stroke/ TIA.

Patients of higher socio-economic status were recruited from urban sites with stroke unit guidelines/protocols and availability of multidisciplinary outpatient teams and private physiotherapy; with $459 / 533$ (86.1\%)

(intervention) and 446/512 (87.1\%)(control) accessing conventional therapy

(medications/rehabilitation) at 6 months.

Primary outcome:

a) $285 / 607$ (47\%) were dead or disabled in the intervention group, $287 / 605$ (47.4\%) in the control (odds ratio $0.98,95 \% \mathrm{Cl}$ 0.78 to $1.23, P=0.87$ )

b) Lack of benefit confirmed with adjusted analysis.

c) One significant interaction by sex of reduced odds of death or dependency in men at 6 months (odds ratio $0.83,95 \% \mathrm{C}$ 0.63 to 1.10 versus odds ratio $1.39,95 \%$ $\mathrm{Cl} 0.93$ to 2.05 for women, $\mathrm{P}=0.04$ for interaction)

Secondary outcomes:

No difference in length of hospital stay (9.3 versus 9.5 days, $\mathrm{P}=0.58$ )

No difference in measures of basic and extended activities of daily living, health-related quality of life, mood and carer strain. 